\title{
Evolution démographique, différenciation et fractures socio-spatiales dans les cantons de Bâle- Ville et Bâle-Campagne
}

Demographic and social change in the Cantons of Basle-City and Basle-Country Bevölkerungsentwicklung und soziale Ausdifferenzierung in Basel-Stadt und Basel-Landschaft

Rita Schneider-Sliwa, Andrea Kampschulte et Daniela Zunzer

\section{revues.org}

Édition électronique

URL : http://rge.revues.org/2770

ISSN : $2108-6478$

\section{Éditeur}

Association des géographes de l'Est

Édition imprimée

Date de publication : 1 janvier 2002

ISSN : 035-3213

Référence électronique

Rita Schneider-Sliwa, Andrea Kampschulte et Daniela Zunzer, «Evolution démographique, différenciation et fractures socio-spatiales dans les cantons de Bâle-Ville et Bâle-Campagne », Revue Géographique de l'Est [En ligne], vol. 42 / 1-2 | 2002, mis en ligne le 04 janvier 2011, consulté le 01 octobre 2016. URL : http://rge.revues.org/2770

Ce document a été généré automatiquement le 1 octobre 2016.

Tous droits réservés 


\title{
Evolution démographique, différenciation et fractures socio- spatiales dans les cantons de Bâle-Ville et Bâle-Campagne
}

\author{
Demographic and social change in the Cantons of Basle-City and Basle-Country \\ Bevölkerungsentwicklung und soziale Ausdifferenzierung in Basel-Stadt und \\ Basel-Landschaft
}

Rita Schneider-Sliwa, Andrea Kampschulte et Daniela Zunzer

\section{NOTE DE L'AUTEUR}

La présente contribution ne peut être exhaustive, compte tenu du volume qui lui est imparti dans ce numéro de la Revue Géographique de l'Est. Elle est ainsi limitée aux aspects essentiels d'une étude détaillée menée sur la structure démographique et la dynamique de la population des deux Cantons de Bâle (Schneider-Sliwa et al., 1999) au moyen des Systèmes d'information géographiques (SIG) et qui a servi de base à la publication d'un atlas - le premier depuis l'atlas structurel de 1967 - sur l'état actuel et les tendances de l'évolution d'un certain nombre d'indicateurs socio-économiques de la région bâloise. Base cartographique (pour l'ensemble des figures) : BFS GEOSTAT/ L+T.

Cartographie : @ Département de Géographie de l'Université de Bâle, géographie humaine, Recherche urbaine et régionale.

1 Les agglomérations apparaissent comme le reflet de la société, car ce sont les lieux sur lesquels la mutation sociale et les problèmes soulevés par elle se manifestent de la façon la plus expressive. Les villes et leur région induisent à la fois la croissance économique et la pauvreté. Ces espaces sont particulièrement significatifs du pluralisme de la société et 
de la densification des problèmes tant sociaux qu'écologiques. Il n'y est plus possible de distinguer les changements intervenus entre les noyaux urbains et la ceinture de l'agglomération. La composante relative à l'occupation du sol n'est plus qu'un aspect certes important, mais tributaire de la problématique de la suburbanisation qui constitue une atteinte à l'écosystème, lui-même lié à des migrations pendulaires croissantes, à l'accroissement de la circulation et des nuisances environnementales. La forte exurbanisation en direction de l'espace suburbain est d'autant plus inquiétante qu'elle amorce souvent un déclin fonctionnel et social de la ville: l'émigration sélective constituée par les catégories démographiques au revenu le plus élevé érode la base fiscale du noyau urbain prédisposé au maintien de la qualité des services en matière culturelle, sanitaire et infrastructurelle. Le transfert démographique s'accompagne également à plus long terme de restructurations ou d'impasses dans le domaine infrastructurel. Les noyaux urbains, grevés par l'accroissement ou le maintien de catégories démographiques âgées ou de groupes marginaux, sont alors placés face à des dépenses particulièrement élevées au point de vue social, sanitaire et infrastructurel. Les transformations sociodémographiques entraînent en fin de compte des coûts sociaux élevés, car la diminution des ménages familiaux et la concentration des problèmes urbains dans certains quartiers induisent de nouveaux processus de ségrégation et des polarisations à l'intérieur du noyau central, ainsi qu'entre la ville et les communes périphériques.

Ces évolutions démographiques générales sont également observées à Bâle depuis la fin des années 1990. La ville de Bâle est certes la troisième ville de la Suisse selon sa taille et forme un important centre économique et culturel à l'échelle confédérale. A ce titre, pour ce qui est de son offre de services et d'emplois, la ville occupe une place supérieure à la moyenne en tant que centre économique régional, eu égard à son niveau de population, quoique depuis 1970 plus de 51000 personnes aient quitté la ville. A cet effet, l'Institut de Géographie de l'Université de Bâle est en train de réaliser, en liaison avec l'office de statistique du canton de Bâle-Ville, un atlas consacré à Bâle et à son espace environnant immédiat. Cet atlas s'appuie sur les Systèmes d'information géographique (SIG) pour illustrer les transformations de la population, de la géographie de l'emploi et de la qualité de vie survenues à Bâle et dans sa région. Cette démarche permet $\mathrm{p}$. ex. de serrer de plus près la mutation des structures socio-économiques dans l'agglomération (fig. 1-3), de même que les processus fonctionnels et sociaux de ségrégation. Elle contribue aussi à appréhender davantage les perspectives spatiales de développement socio-économique. La présente contribution donne un aperçu des développements socio-démographiques à Bâle et de leurs répercussions. 
Figure 1 : Délimitation et structure interne de l'agglomération de Bâle, 1998

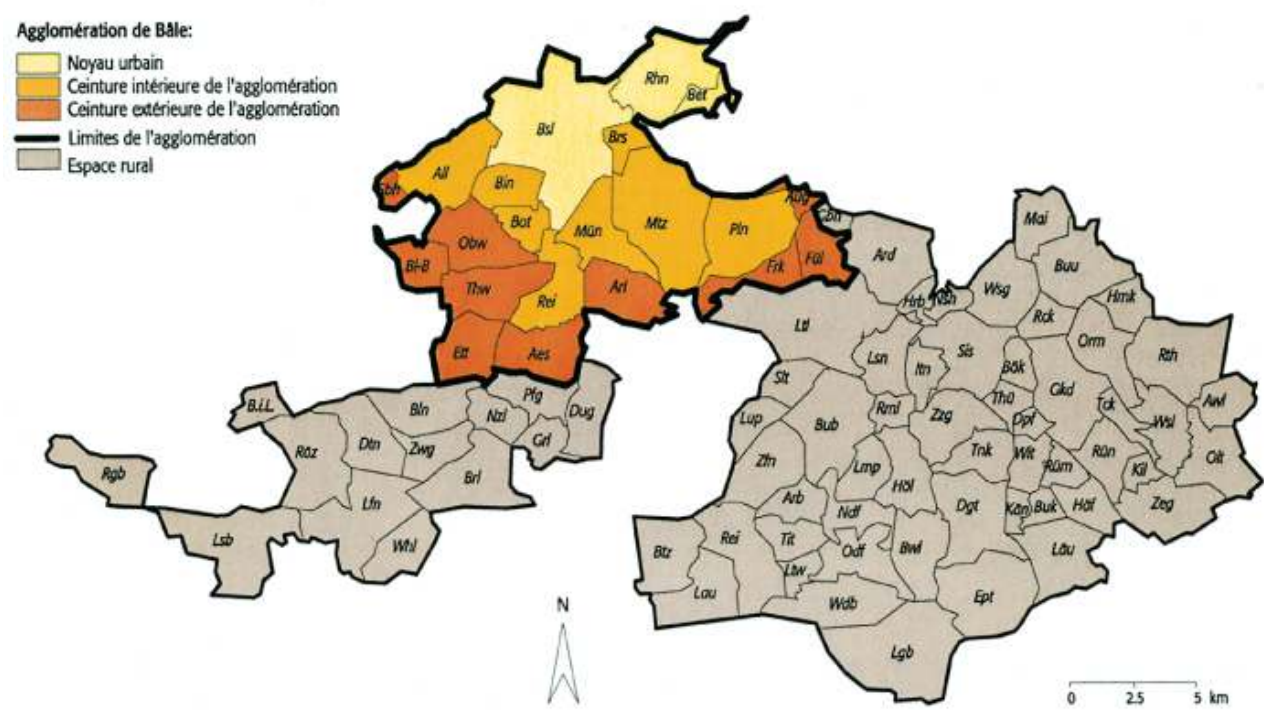

La figure distingue trois unités spatiales principales dans l'agglomération : le noyau urbain, ainsi que les ceintures intérieure et extérieure. Les subdivisions du noyau urbain sont les quartiers, celles des deux cantons de Bâle (Bâle-Ville et Bâle-Campagne) les composantes communales.

Figure 2 : Quartiers résidentiels du canton de Bâle-Ville

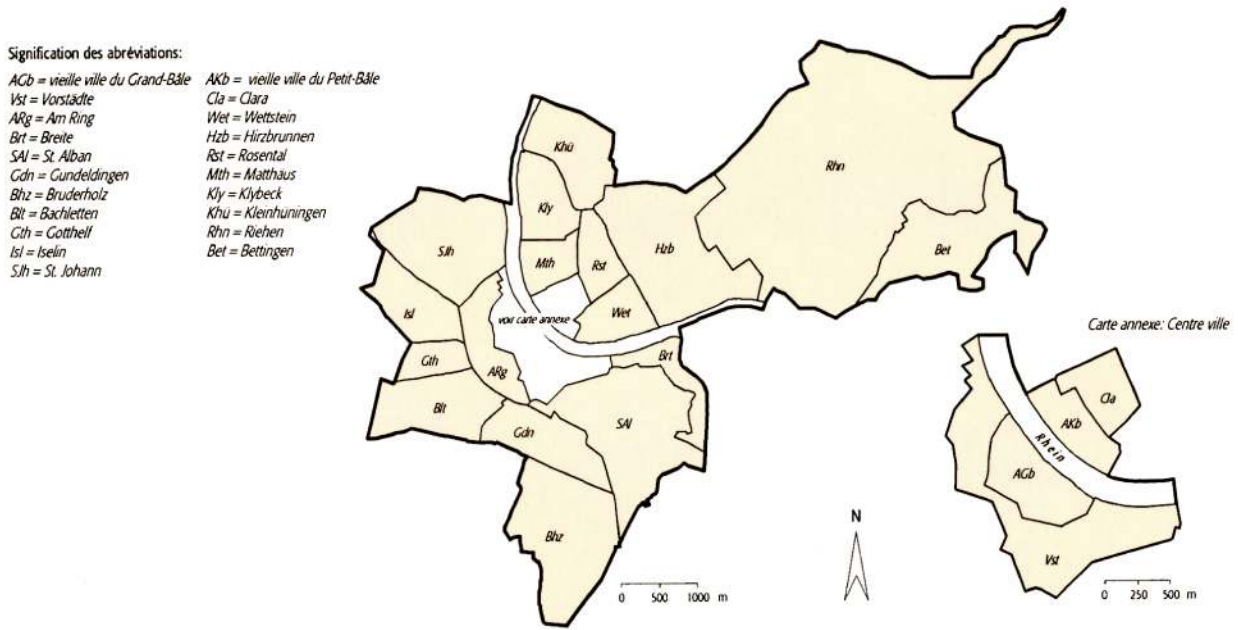




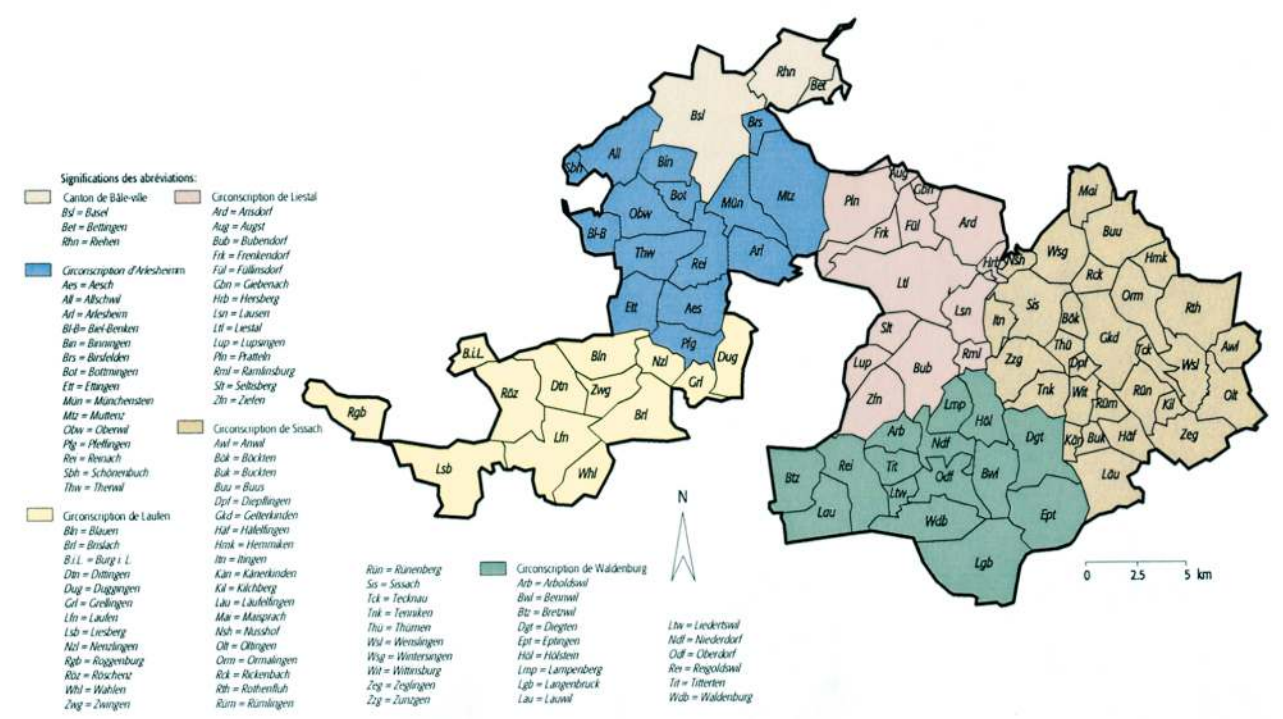

Les données statistiques utilisées relèvent essentiellement du recensement de la population de 1990 ou des dernières données intercensitaires disponibles jusqu'en 1999. La publication de l'ensemble des résultats du recensement de l'année 2000 est annoncée pour l'année 2003. Les premiers résultats présentés lors de la conférence de presse du 22 janvier 2002 par l'Office statistique fédéral ne confirment toutefois pas seulement les tendances signalées dans le présent article, mais aussi l'accélération de la périurbanisation. Ils révèlent que l'évolution en cours est engagée dans le sens contraire des objectifs fixés à l'aménagement suisse, et continue à alimenter la problématique soulevée par la ville (Basler Zeitung, 23.1.2002, pp.1 et 2).

\section{L'évolution démographique dans la région bâloise}

\section{A. Aperçu général}

Depuis le début du XX $\mathrm{XX}^{\mathrm{e}}$ siècle, la population suisse a augmenté régulièrement. De 1941 à 1970, cette croissance l'a fait passer de 4,3 millions à 6,3 millions d'habitants, notamment par suite de l'immigration étrangère. En 1999, la Suisse compte 7,16 millions d'habitants ( Annuaire statistique de la Suisse, 2001). Jusqu'en 1960, l'évolution de la population de la ville de Bâle a progressé de façon analogue à celle de la Suisse dans son ensemble. Celle de Bâle-ville a doublé : de 112200 habitants en 1900, elle est passée à 225600 en 1960. Durant la décennie 1960-1970, l'augmentation de la population de Bâle est certes demeurée en retrait par rapport à celle de la Suisse en général, mais elle a néanmoins progressé, pour atteindre 235000 habitants. Celle du canton de Bâle-Ville diminue toutefois depuis les années 1970 : elle ne compte plus que 234900 habitants en 1970 et 191800 en 1999 (Tableau 1). 
Tableau 1 : Evolution de la population (en milliers d'habitants) en Suisse et dans les Deux-Bâles (1900-1999)

\begin{tabular}{|c|c|c|c|}
\hline Année & Suisse & Bâle-Ville & Bâle-Campagne \\
\hline 1900 & 3315,4 & 112,2 & 68,5 \\
1910 & 3753,3 & 135,9 & 76,5 \\
1920 & 3880,3 & 140,7 & 82,4 \\
1930 & 4066,4 & 155,0 & 92,5 \\
1941 & 4265,7 & 170,0 & 94,5 \\
1950 & 4715,0 & 196,5 & 107,5 \\
1960 & 5429,1 & 225,6 & 148,3 \\
1970 & 6269,8 & 234,9 & 204,9 \\
1980 & 6366,0 & 203,9 & 219,8 \\
1990 & 6873,7 & 199,4 & 233,5 \\
1999 & 7164,4 & 191,8 & 261,4 \\
\hline
\end{tabular}

Source : Recensements fédéraux ; Office fédéral de la statistique, 2001 ; Statistisches Amt Kanton Basel-Lanschaft, 2001 ; Statistisches Amt Kanton Basel-Stadt, 2001

La décroissance de Bâle-Ville a été compensée par la croissance de Bâle-Campagne. De 1950 au milieu des années 1990, la population de Bâle-Campagne est devenue 2,5 fois plus importante (107 500 habitants en 1950, 256500 en 1996); durant les seules années 1950-1970, elle a presque doublé. Quoique après 1970 la croissance ait diminué, les taux de croissance sont restés nettement au-dessus des taux fédéraux. Parallèlement à l'émigration survenue dans le canton de Bâle-Ville, cette croissance a été sous-tendue de façon déterminante par l'immigration de travailleurs étrangers. Dans le cadre de BâleCampagne, la population étrangère a sextuplé de 1950 à 1970, progressant de 6600 à 38600 habitants.

Ces évolutions se sont poursuivies dans les années 1990: la population helvétique a continué à progresser, quoique de façon relativement lente. Ce fut aussi le cas à BâleCampagne. Dans la ville de Bâle, même après quelques courtes séquences de progression, la régression reprit dès 1993 et passa à la fin des années 1990 au-dessous du niveau de 1950. L'administration publique s'attend pour les prochaines années à la poursuite de la récession démographique à Bâle-Ville; pour Bâle-Campagne elle prévoit la poursuite d'une légère croissance. 


\section{B. Evolution démographique micro-spatiale à Bâle et dans son espace environnant}

7 Les composantes micro-spatiales de Bâle-Ville et Bâle-Campagne révèlent toutefois une évolution démographique très différenciée par rapport aux données exposées ci-dessus.

8 La figure 4montre que le début de la décroissance démographique, durant les années 1970, entraîna d'abord des pertes de population dans l'ensemble des quartiers de la ville de Bâle (avec une croissance maximale de 3,4 \%). Ce n'est que durant les années suivantes que l'évolution se différencia à l'échelle micro-spatiale. Bâle-Campagne accusa durant les années 1970 des contrastes spatiaux accusés (fig. 7). Sa population s'est accrue de $7 \%$ durant les années 1970 ; certaines communes ont cependant perdu des habitants : Cette récession frappa d'une part les communes localisées à proximité immédiate du noyau urbain, d'autre part les communes très périphériques dépourvues de voies de circulation adéquates. La croissance la plus forte eut lieu dans les communes rurales bénéficiant d'une bonne accessibilité à la ville. La ceinture de l'agglomération (fig. 2 et 3) localisée autour du noyau urbain connut une forte croissance durant cette décennie.

Figure 4 : Evolution démographique de Bâle-Ville (1970-1980)

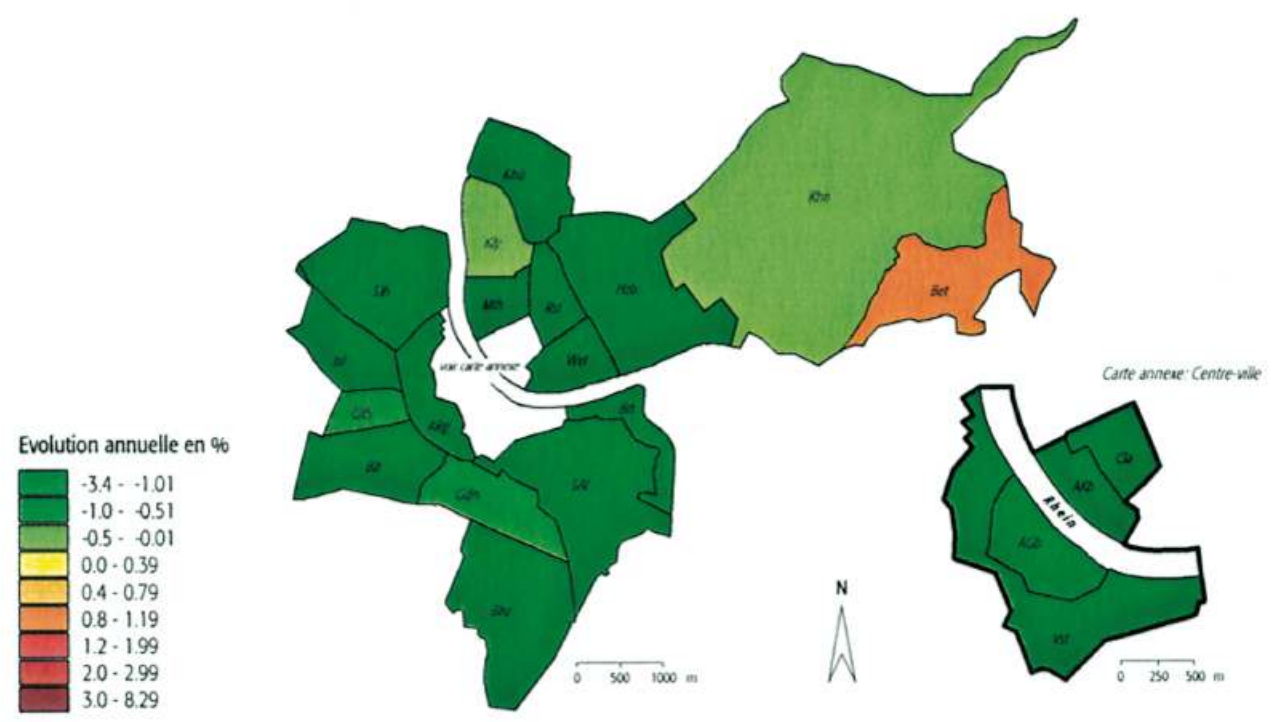

Dans les années 1980, la ville de Bâle connut une régression démographique de 2,2 \% (fig. 5). Comme sur les deux rives du Rhin se manifesta une légère croissance dans les divers quartiers du centre-ville, une ségrégation socio-spatiale apparut à l'échelle microspatiale. A l'exception de la vieille ville, dotée d'avantages résidentiels, il s'agit là avant tout de quartiers d'habitation dont le taux d'habitants non-suisses est élevé. Par là-même les causes de cet apport sont très différenciées. Pour ce qui est des apports en vieille ville, dans les logements rénovés, il pourrait s'agir de ménages acquis au retour vers le centre en raison de l'attrait de la «nouvelle urbanité ». Dans les autres quartiers, la croissance pourrait s'expliquer par une évolution naturelle plus rapide que celle propre à la population dans son ensemble, et par une augmentation de la population d'origine étrangère. 
Evolution annuelle en $\%$

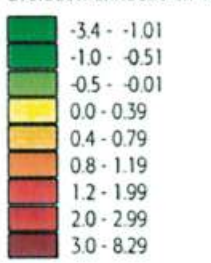

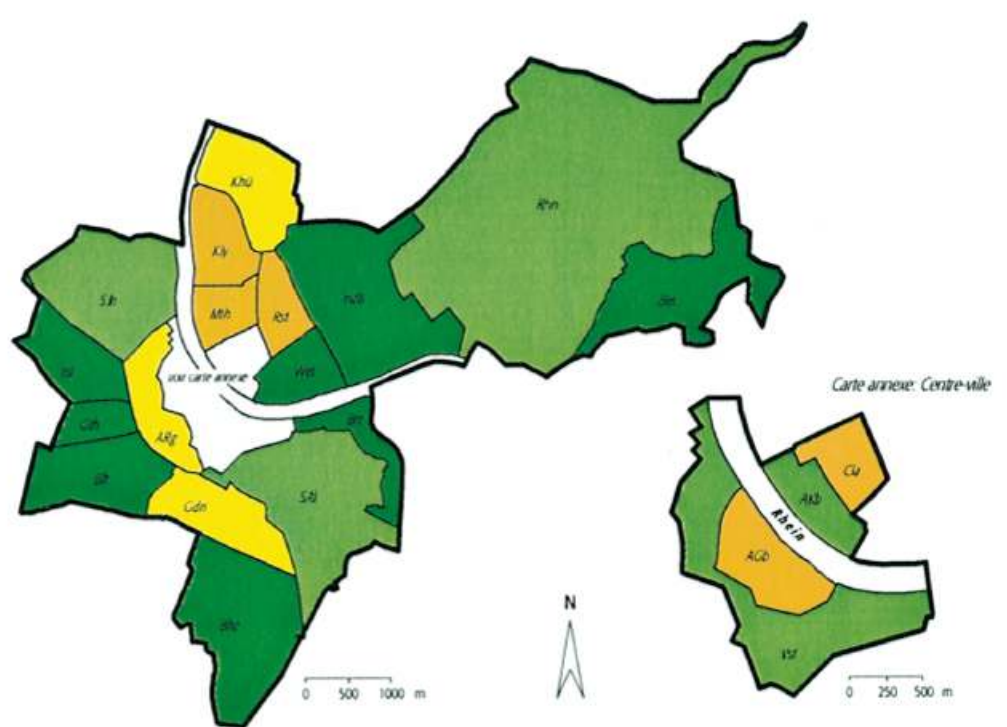

le sens d'une émigration successive vers l'espace périurbain et vers les communes extraurbaines (fig. 10). Durant les années 1980, les communes jouxtant la ville ont subi de légères pertes de populations, voire une certaine stagnation (fig. 8); les communes restantes connurent une croissance. La croissance la plus forte s'est déplacée de plus en plus vers les communes périphériques restantes, notamment depuis les centres de Bâle et de Liestal, en direction des communes demeurées rurales.

Figure 6 : Evolution démographique de Bâle-Ville (1990-1996)

Evolution annuelle en $\%$
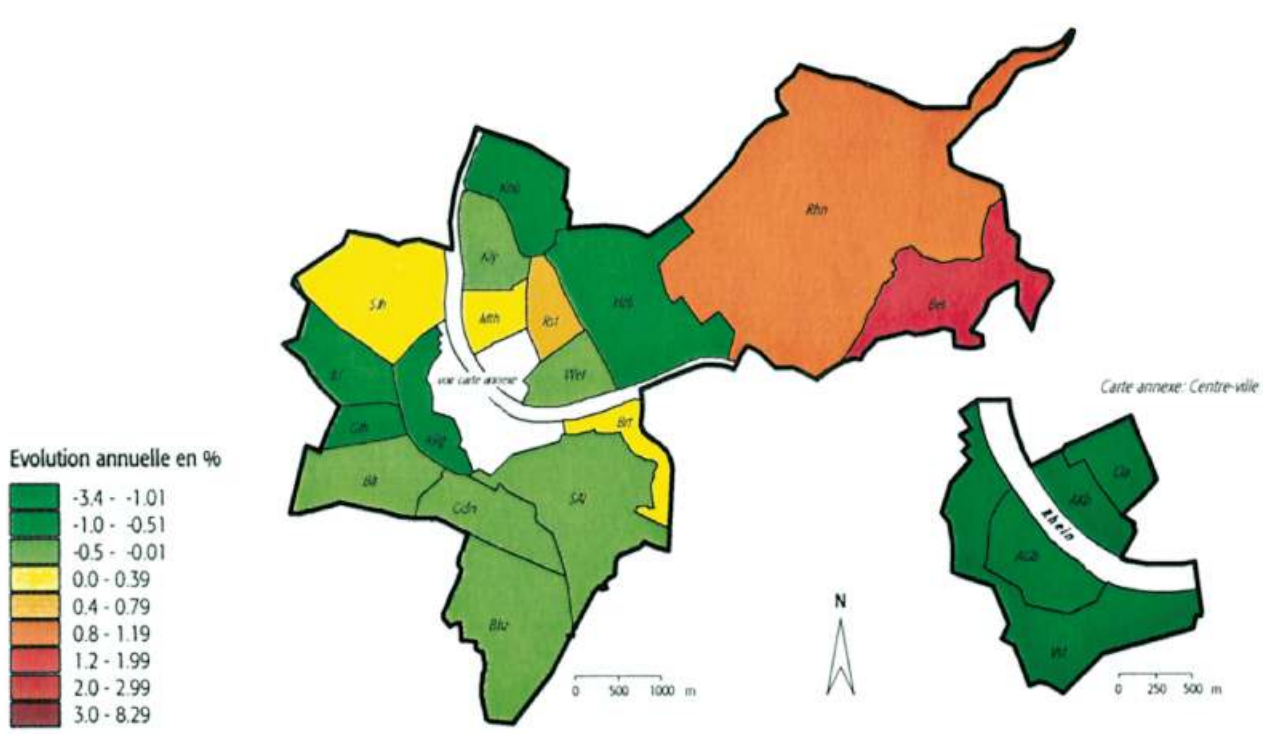

Durant les années 1990, les secteurs surtout marqués par un cachet de petite ville dans le canton de Bâle-Ville, tels que ceux de Riehen et Bettingen, ainsi que quelques « quartiers ouvriers » ou quartiers d'immigrés (p. ex. Sankt-Johann, Matthäus, Rosenthal) 
ont vu augmenter leur population (fig. 6).La vieille ville par contre a perdu en population. La poursuite de l'augmentation démographique dans les communes rurales de Bettingen et Riehen s'explique entre autres par la poursuite de l'activité dans le bâtiment sur des réserves foncières, ainsi que par le fait que les deux communes témoignent d'un niveau élevé de qualité résidentielle par suite de leur proximité par rapport à la ville. Elles bénéficient en outre d'une image particulièrement positive, compte tenu des taux d'imposition fiscale modérés au regard de l'ensemble cantonal. A Bâle-Campagne la ceinture intérieure de l'agglomération a continué à décliner, tandis que les communes périphériques ont gagné en habitants (fig. 9).

Figure 7 : Evolution de la population dans les Deux-Bâles (1970-1980)

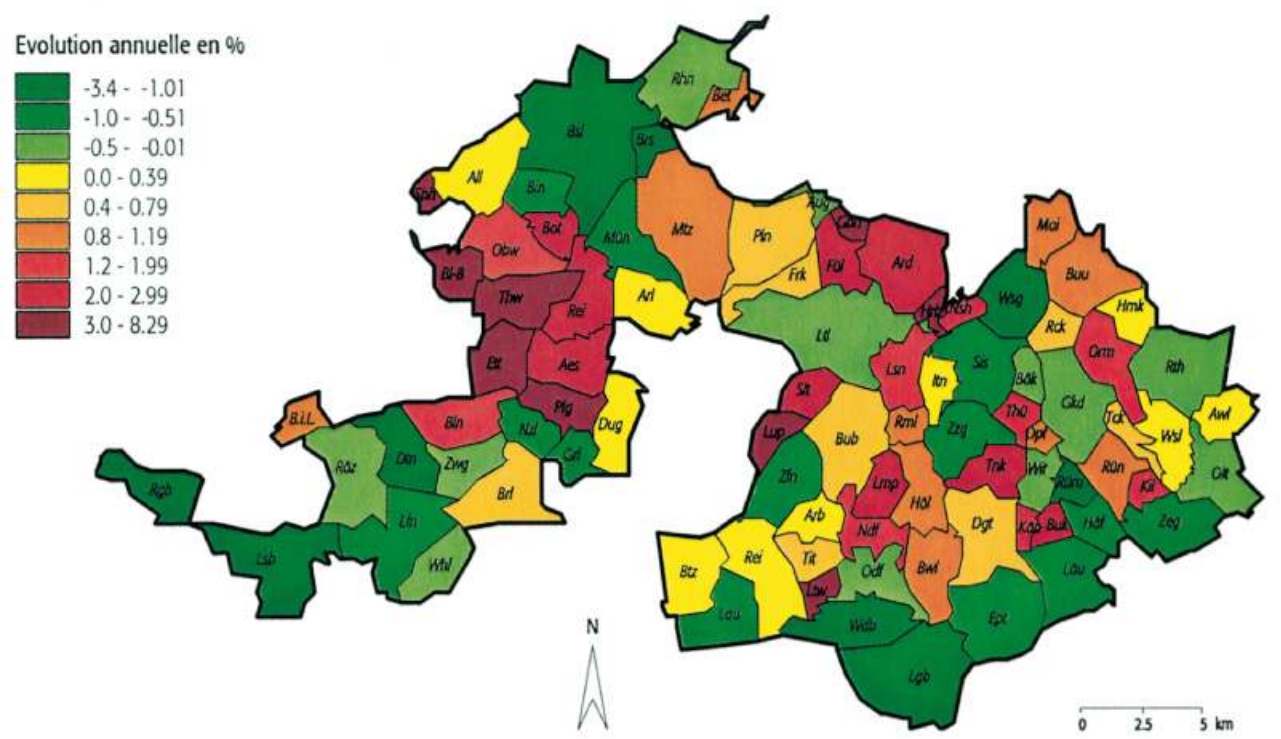

Figure 8 : Evolution de la population dans les Deux-Bâles (1980-1990)

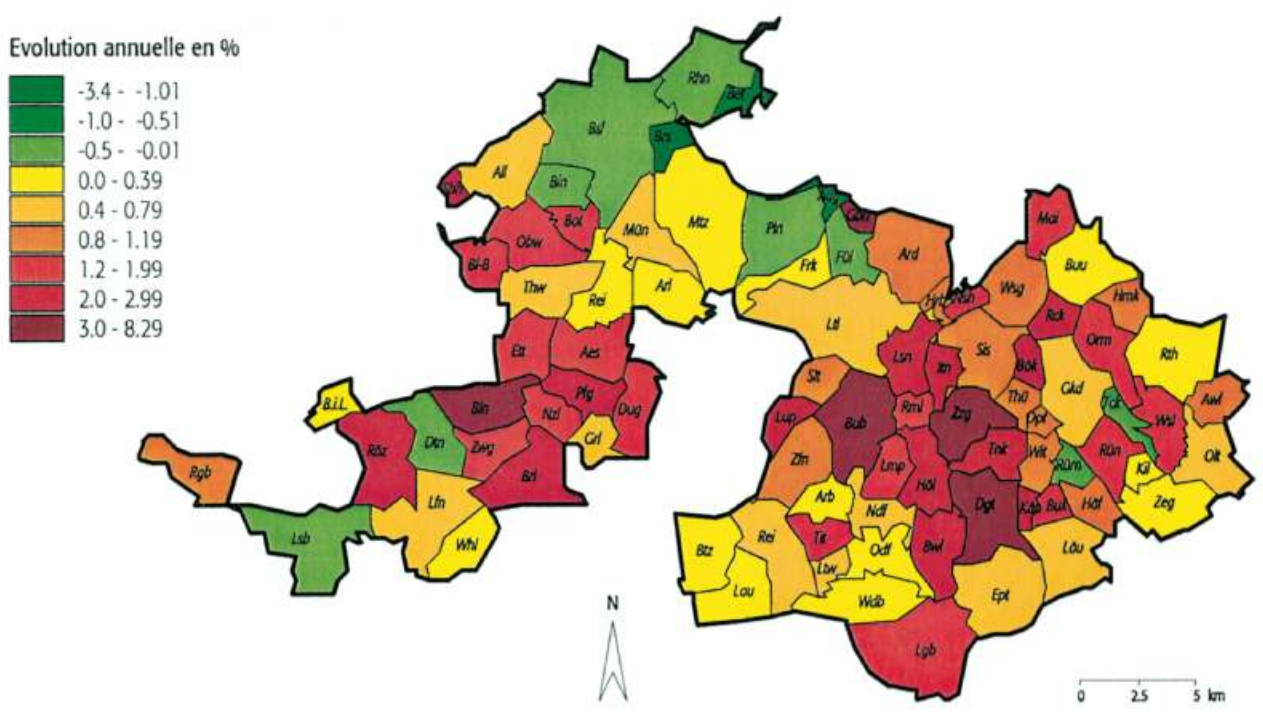


12 Une analyse fine de l'évolution et de la répartition démographiques opérée à l'échelle micro-spatiale depuis les années 1970 révèle ainsi qu'après des pertes initiales, certaines parties de la ville de Bâle ont de nouveau gagné en habitants. Il s'agit notamment de localisations résidentielles attractives en centre-ville et en aire périphérique, de même que de quartiers à taux élevé d'immigrés d'origine étrangère. Autour de Bâle, la zone de plus grande croissance démographique s'éloigne de plus en plus du noyau urbain depuis les années 1970. Si la tendance actuelle se poursuit, la ville et l'aire qui la jouxte doivent compter avec la poursuite de la réduction de la densité démographique; dans les communes rurales, par contre, celle-ci tend à augmenter.

Figure 9 : Evolution de la population dans les Deux-Bâles, 1990-1996

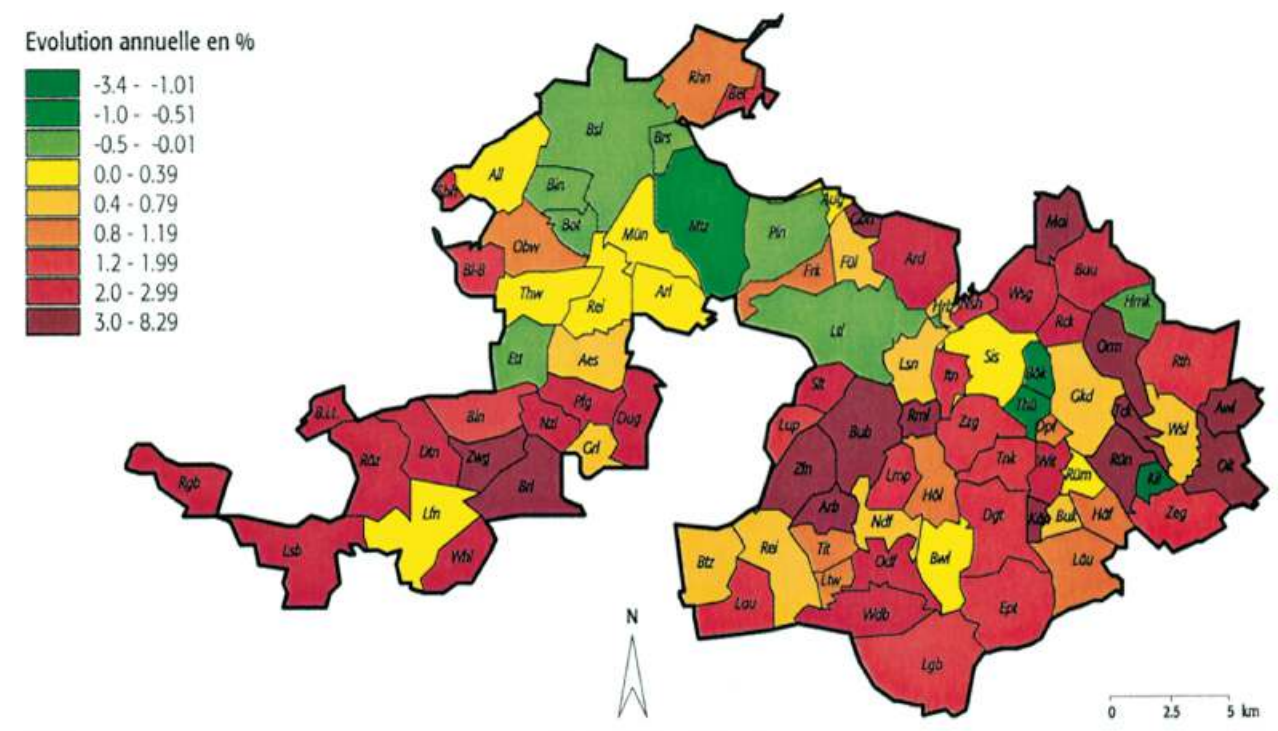


Figure 10 : Migration successive dans les communes péri-urbaines et non-urbaines de l'agglomération de Bâle, de 1970 à ce jour

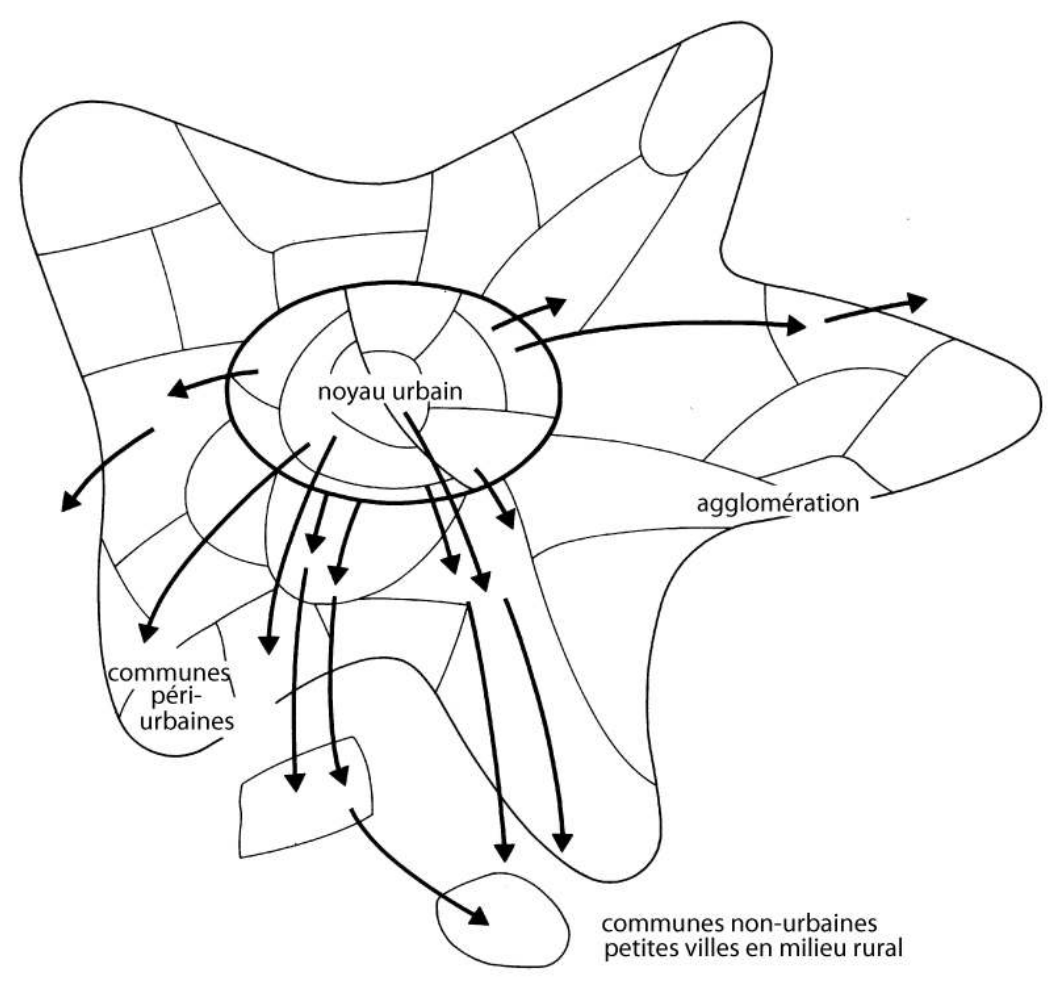

\section{L'évolution socio-économique de la structure démographique}

Les modifications démographiques d'une région de grande ville comme celle de Bâle ne se limitent pas au façonnement d'une nouvelle répartition résidentielle. Elles impliquent aussi une transformation des structures de la société et de la façon de vivre. Les structures familiales «classiques» se dissolvent toujours davantage en de multiples nouvelles manières de vivre et de formes de ménages appelées «nouveaux types de ménages ». Dans les grandes villes, le nombre de ménages de taille réduite augmente par rapport à celui des ménages familiaux, surtout sous la forme de couples sans enfants (« double income no kids» = Dinks) ou sous celle de personnes sans enfants au revenu cossu - une forme choisie délibérément en vue d'une vie aussi indépendante que possible. Le lien confessionnel des ménages est plutôt en train de diminuer. Il convient de comparer la réduction spectaculaire du nombre de ménages à lien confessionnel durant les années 1980 avec les modifications du droit fiscal (surtout la séparation de la perception de l'impôt pour les Eglises chrétiennes de l'impôt général) pour constater que l'instauration de la liberté de versement de l'impôt pour l'Eglise a conduit à de nombreuses décisions individuelles de quitter l'Eglise.

Les processus de ségrégation sociale et l'individualisation des comportements révèlent de façon plus nette qu'ailleurs les différences micro-spatiales et les évolutions parallèles apparemment contradictoires, telle que la poursuite de la suburbanisation en même 
temps que les tendances à la réurbanisation. Nous proposons de livrer ci-après les observations recueillies à ce sujet dans la région bâloise.

\section{A. Les structures d'âge}

La diminution du nombre d'enfants ainsi que l'élévation de l'espérance de vie sont des phénomènes dominants dans les sociétés occidentales. Alors que le nombre d'enfants diminue, le taux des personnes âgées augmente. A Bâle-Ville et Bâle-Campagne, le taux des plus de 64 ans augmente depuis les années 1960 de manière plus significative dans le canton urbain que dans le canton rural.

Une comparaison des structures démographiques entre Bâle-Ville et Bâle-Campagne (fig. 11) révèle que celles propres à la population suisse se différencient nettement de celles de la population étrangère. Des différences marquantes sont à signaler aussi entre les deux cantons. La périphérie accuse dans l'ensemble un taux d'enfants et d'adolescents plus élevé, de même que d'adultes à l'âge actif, tandis que la part de la population âgée dans le noyau urbain est supérieure à celle de l'espace ambiant.

Figure 11 : Pyramides des âges de Bâle-ville et de Bâle-Campagne selon la nationalité et le sexe, 1996

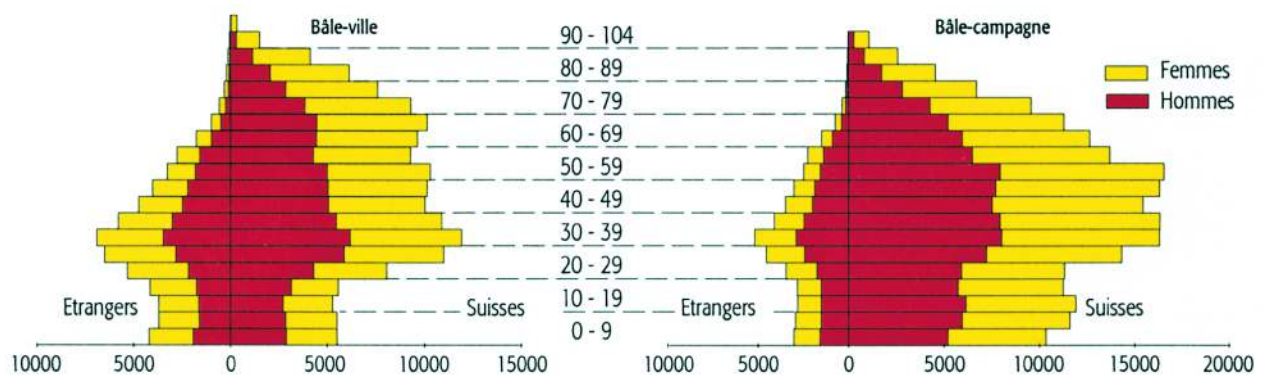

Dans la population étrangère, le taux d'enfants et d'adolescents est comparativement élevé et celui de la population retraitée très faible. Ce phénomène s'explique tout spécialement par la durée de séjour des travailleurs migrants. Les anciens travailleurs immigrés commencent seulement à devenir progressivement des retraités pensionnés. Certains retournent à ce moment-là dans leur pays d'origine. D'autres ont été ait naturalisés entre temps et ne relèvent plus de la statistique des étrangers.

A l'échelle des quartiers d'habitation, voire des communes (fig. 12) des différences microspatiales apparaissent nettement. Certains de ces quartiers accusent des particularités structurelles en matière démographique. Le cas des contrastes très nets dans les deux quartiers bâlois voisins de Gundeldingen et Sankt-Alban est exemplaire. A Gundeldingen les taux de population étrangère et de jeunes adultes tant Suisses que nonSuisses sont élevés. Alors que la part des enfants et adolescents est plus faible dans la population suisse que dans la population étrangère, celle-ci a un taux de population âgée plus faible. Les taux élevés de population étrangère et de jeunes adultes témoignent du fait que le quartier du "Gundeli» (Gundeldingen) comporte aussi des possibilités de logements pour des habitants ne disposant que d'un revenu faible, inférieur à la moyenne bâloise. Dans le quartier voisin de Saint-Alban, la part de population étrangère est plus réduite et le vieillissement de la population dans son ensemble accusé. Près de $30 \%$ des 
habitants y ont plus de 64 ans. Il s'agit là d'un quartier résidentiel traditionnel comportant une population suisse à revenu élevé.

Figure 12 : Pyramides des âges par quartiers résidentiels et communes à Bâle-Ville, 1996

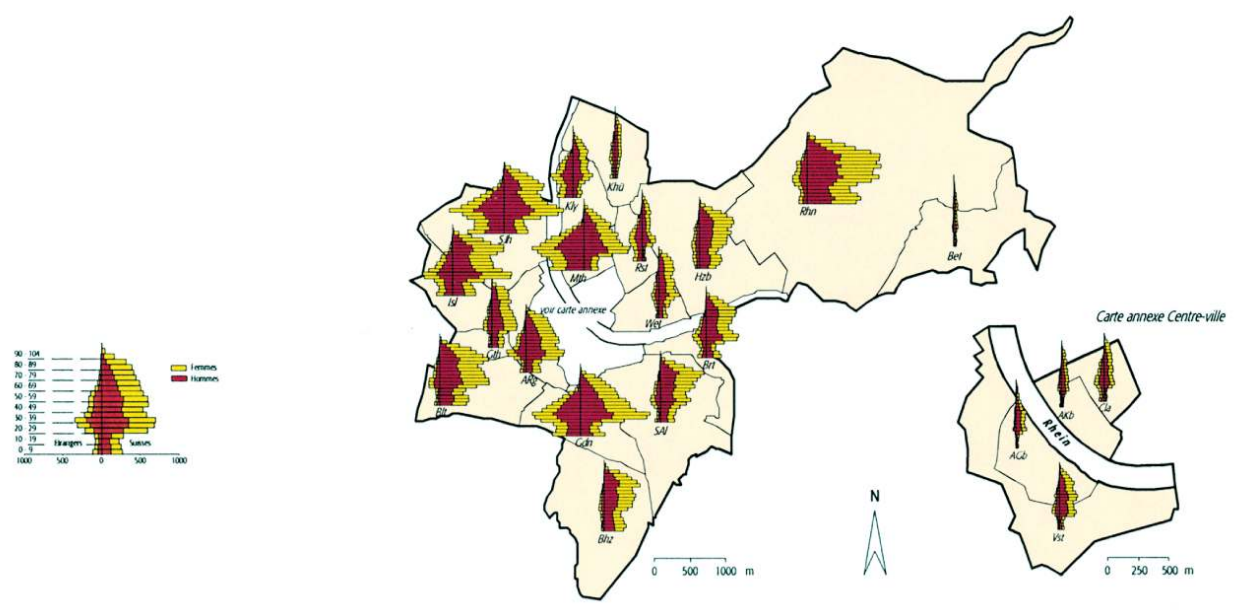

Des différences micro-spatiales analogues peuvent être relevées également à $B a ̂ l e-$ Campagne. Les communes suburbaines typiques de l'agglomération qui ont connu une forte croissance au cours des dernières décennies par suite de l'arrivée de personnes actives en provenance du noyau urbain, ne comptent qu'un faible taux de population étrangère, mais un pourcentage élevé d'actifs par rapport à celui des enfants et adolescents (p. ex. Reinach).

C'est en ville que la part des enfants par rapport à la population totale est la plus faible. Elle augmente dans les secteurs périurbains typiques. Dès lors, cependant que ces secteurs sont localisés à proximité de la ville, comme à Reinach, nous observons déjà un phénomène de vieillissement démographique (fig. 13 et 14).Dans les communes de l'agglomération proches de la ville, le taux d'enfants est plus faible que la moyenne de Bâle-Ville (de 13-15\% en comparaison avec une moyenne de $16 \%$ ). Cette situation peut s'expliquer par une appartenance de ces communes à la première vague de suburbanisation. Ici les enfants, devenus entre-temps adultes, ont suscité le phénomène de l'« empty nest » en délaissant le foyer familial. Cette évolution n'est toutefois pas celle de la totalité des communes situées à l'intérieur de la ceinture de l'agglomération. L'exemple de Pratteln montre qu'il peut en être tout autrement, dans la mesure où cette commune est moins une commune-dortoir suburbaine qu'une commune industrialotertiaire aux nombreux emplois. La part de population étrangère et celle des enfants ou adolescents y est manifestement supérieure. 


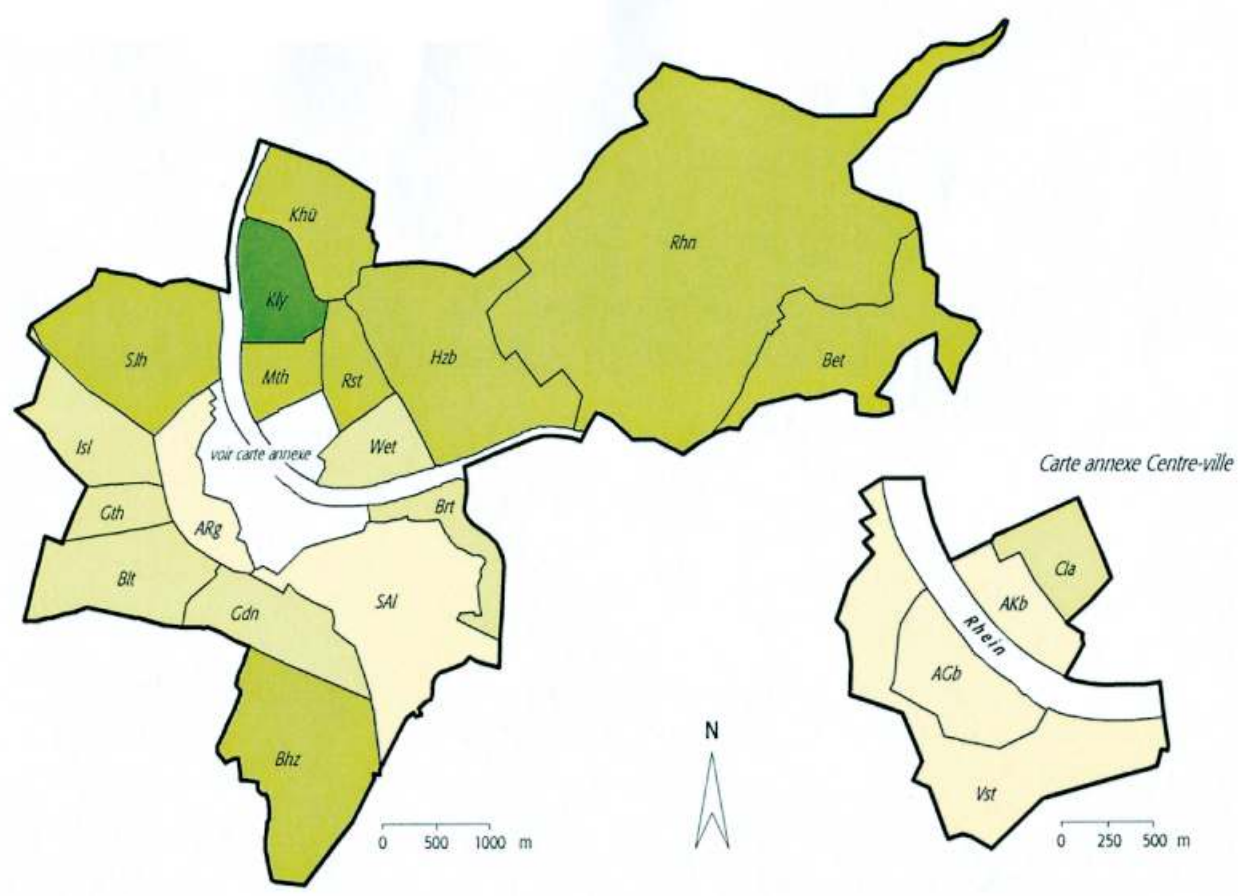

Figure 14 : Taux d'enfants (pourcentage de moins de 15 ans) dans les Deux-Bâles, 1996

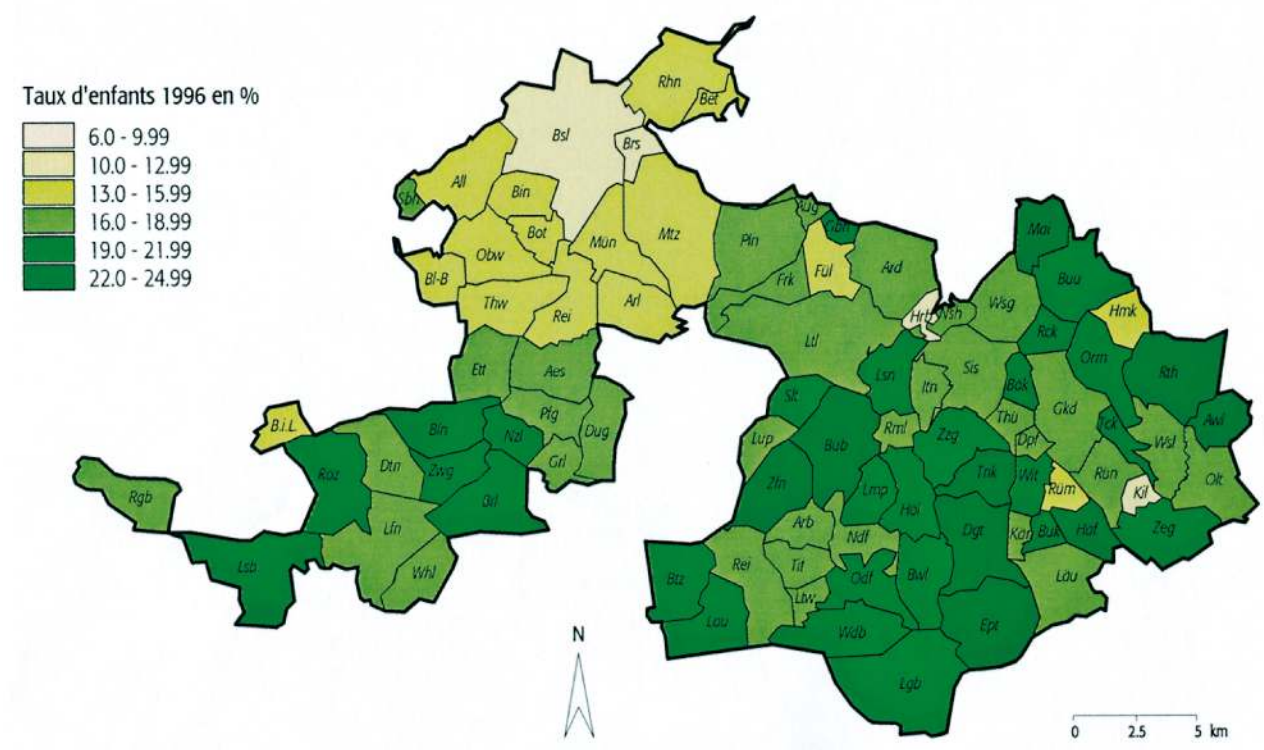

Dans les communes rurales jouxtant l'agglomération, de nombreuses familles jeunes ainsi que des familles en voie de constitution se sont établies au cours des toutes récentes années. C'est la raison pour laquelle les communes rurales au taux de croissance le plus élevé depuis les années 1980 se caractérisent aussi par les plus forts pourcentages d'enfants et d'adolescents. Le noyau urbain est marqué de façon très nette par les taux d'enfants les plus faibles. A l'intérieur même de la ville, c'est de nouveau la vieille ville qui connaît les taux d'enfants les moins élevés. 
Le taux de population âgée est inversement proportionnel à celui des enfants et adolescents (fig. 15 et 16).Il existe très clairement un décalage entre la ville, qui enregistre les valeurs les plus élevées, et les autres communes de l'agglomération; les communes rurales n'ont en fin de compte qu'une proportion très faible de personnes âgées. Celles-ci atteignent par contre les taux les plus élevés en ville, dans les quartiers des classes moyennes et supérieures ; les secteurs à population étrangère importante ont les taux de personnes âgées les plus réduits.

Figure 15 : Taux de personnes âgées (pourcentage de plus de 64 ans) à Bâle-Ville, 1996

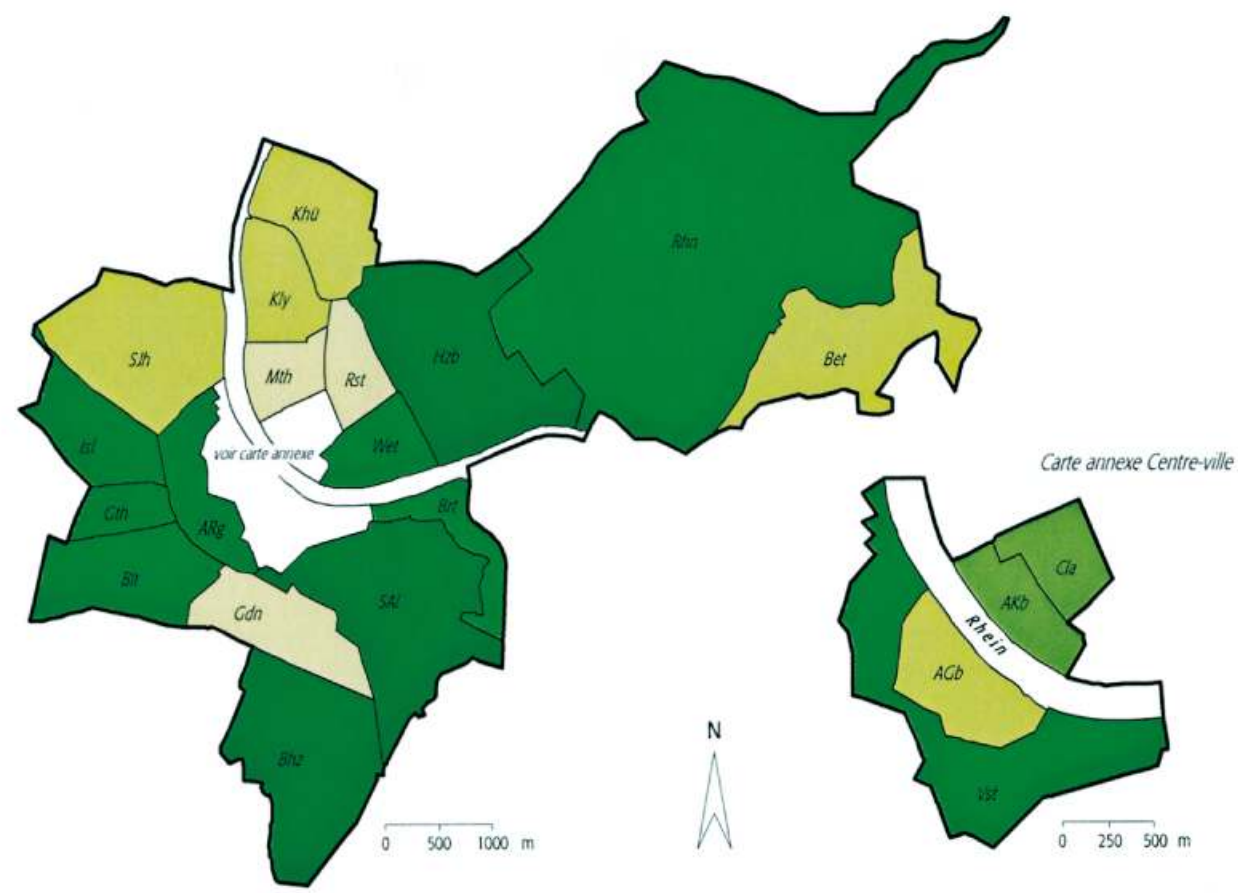

Figure 16 : Taux de personnes âgées (pourcentage de plus de 64 ans) dans les Deux-Bâles, 1996

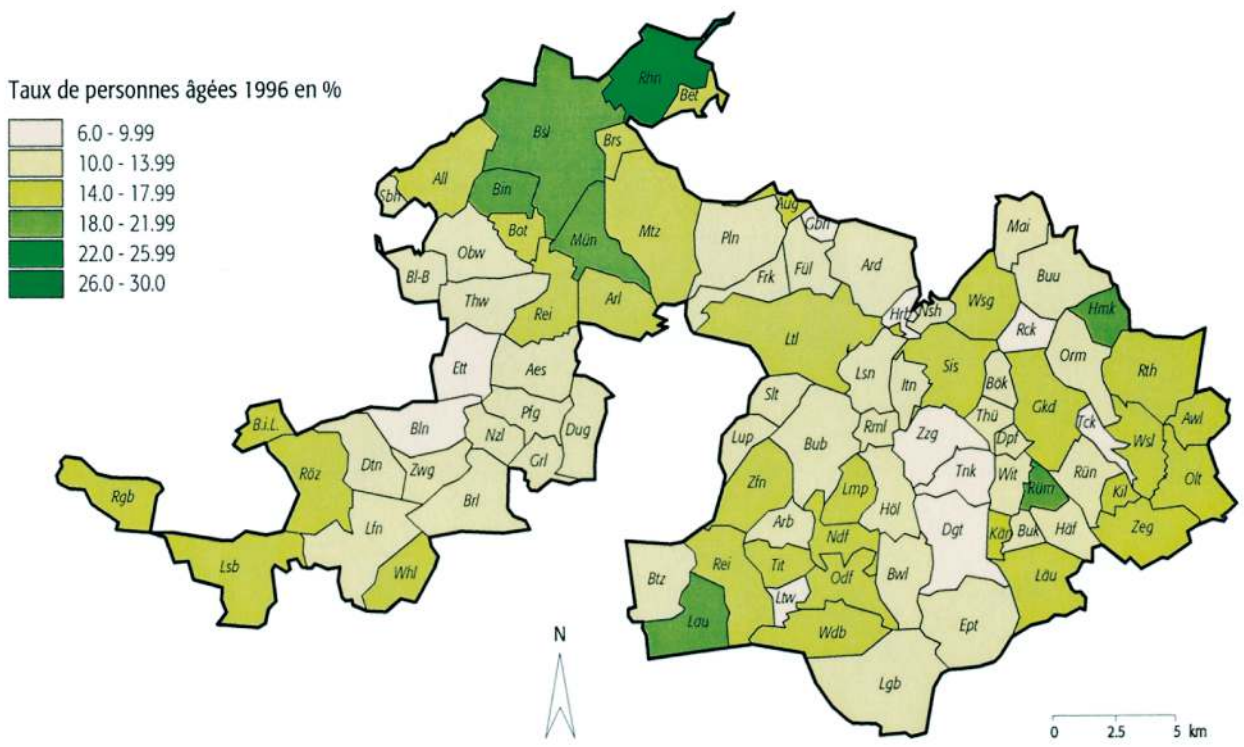




\section{B. La répartition résidentielle des populations suisse et étrangère} nombre d'étrangers à Bâle a presque doublé entre 1960 et 1970, soit 19400 en 1960 et 37700 en 1970. En 1996, Bâle comptait 52200 étrangers, un chiffre qui correspondait à 27 \% de la population. Dans le canton de Bâle-Campagne, 17 \% de la population n'avait pas de passeport suisse.

En ce qui concerne les lieux de résidence de la population étrangère, la ville de Bâle est marquée par deux secteurs principaux. Il s'agit d'une part des quartiers d'habitation du nord-ouest, localisés de part et d'autre du Rhin : Matthäus (50\%), Klybeck (46\%), SanktJohann (41 \%), Kleinhüningen (39\%), Altstadt Kleinbasel - vieille ville de Petit-Bâle - (25 \%). Le quartier de Gundeldinger forme, avec $37 \%$ de population étrangère, l'autre secteur. Les taux d'étrangers les plus faibles sont propres à Bachletten, à Bruderholz et à Riehen, c'est-à-dire aux communes réservées aux catégories moyennes et supérieures de la population. La population étrangère habite ainsi essentiellement dans les quartiers résidentiels à forte activité industrielle et tertiaire, grevés de surcroît par de fortes nuisances induites par la circulation. C'est aussi dans ces quartiers que la proportion de vieux logements non rénovés est particulièrement élevée, situation qui tend à réduire la qualité résidentielle.

Dans la ville de Bâle, la répartition différenciée des nationalités est toutefois significative de contrastes entre résidents étrangers. Une étude comparée effectuée dans plusieurs villes suisses (Wimmer, 2000) a montré que le choix résidentiel est fonction du type de nationalité étrangère et qu'il existe un lien entre le statut social du résident et son lieu d'habitation. La population non suisse semble néanmoins se répartir en deux catégories: "les nationalités d'Europe du Sud, turque et ex-yougoslave, de même que celles du Tiers-Monde d'une part, celles d'Europe du Nord, du Canada et des USA d'autre part » (Wimmer, 2000). Le lieu d'habitation de la population étrangère semble donc être souvent tributaire de la qualification et de la nature de l'activité, mais aussi de la durée du séjour. Alors que les immigrés recherchent fréquemment des logements à proximité d'autres compatriotes parlant la même langue, cette forme de concentration diminue au fur et à mesure qu'augmente le degré d'intégration à la société d'accueil. Quoique les quartiers d'habitation dont le taux de population étrangère dépasse $20 \%$ comportent le plus fréquemment des ressortissants italiens, ex-yougoslaves et turcs, la population italienne, au regard des deux autres types de nationalité, est tout de même répartie de manière très uniforme sur l'ensemble du territoire urbain. Dans les quartiers résidentiels qui n'accusent qu'un faible taux moyen d'étrangers, la part des ressortissants d'Europe septentrionale et centrale est élevée. Ces derniers sont toutefois répartis de façon relativement uniforme dans l'ensemble de la ville (Eder, 2001).

$\mathrm{Au}$ regard de la répartition spatiale de la population étrangère dans le canton de BâleCampagne, il existe un important décalage entre les communes urbaines et les communes rurales. Dans les communes situées à l'intérieur et à l'extérieur de la ceinture de l'agglomération de Bâle, le nombre d'étrangers est certes plus élevé que dans les communes rurales du canton; le taux d'étrangers par contre est cependant relativement réduit - 13 à $16 \%$ - dans de nombreuses communes, comme celles de Muttenz, Oberwil ou Bottmingen. Habiter ici coûte trop cher pour une grande part de la population étrangère et la prépondérance des maisons monofamiliales qui rendent ces communes très typiques

Revue Géographique de l'Est, vol. 42 / 1-2 | 2010 
y favorise les catégories moyennes et élevées de la population. La question demeure cependant posée - non seulement à propos de la ville de Bâle - de savoir pourquoi les ressortissants d'origine italienne, en tant que catégorie d'étrangers à très longue durée de séjour en Suisse, ne participent pas ou guère au processus de suburbanisation, contrairement au comportement des ressortissants d'Europe du Nord et d'Europe centrale qui, eux, y participent toujours davantage.

\section{Taille des ménages et types de ménages}

\section{La taille des ménages}

Le nombre de ménages a presque doublé en Suisse de 1960 à 1990, alors que la population n'a augmenté que de $27 \%$. L'essentiel de cette augmentation numérique des ménages s'explique d'abord par la réduction de leur taille. La part des ménages d'une personne, voire de deux personnes, s'est sensiblement accrue au cours des dernières décennies. Par contre celle des ménages de quatre personnes ou plus a diminué. En 1990, la moyenne des ménages à une personne s'est établie autour de $32 \%$ pour l'ensemble de la Suisse.

Tandis que l'évolution dans le canton de Bâle-Campagne s'est déroulée de façon générale parallèlement à celle de la moyenne suisse, celle de Bâle-Ville s'en est distanciée par un accroissement de plus du double du taux des ménages d'une personne. Dans le canton de Bâle-Ville, la diminution de la taille des ménages a débuté plus tôt ; cette réduction est à présent plus prononcée qu'en territoire rural. La part des ménages d'une personne, déjà de $21 \%$ en 1960, s'est élevée à $45 \%$ en 1990.

Dans la ville de Bâle, des différences sensibles apparaissent à l'échelle des quartiers. En 1990 le taux de ménages d'une personne s'y situe en moyenne autour de $47 \%$. Ce taux atteint 55 à $60 \%$ dans les secteurs attractifs de la vieille-ville du Grand-Bâle (Altstadt Grossbasel) et de celle du Petit-Bâle, ainsi que dans les villes de banlieue. Même dans le quartier de Gundeldingen, un ancien quartier ouvrier à présent très apprécié par les étudiants, situé à proximité immédiate de la gare, le taux de ménages d'une personne atteint tout juste $50 \%$. Ce taux diminue progressivement au fur et à mesure que l'on se rapproche de la périphérie de la ville. Ce décalage entre le noyau et la périphérie est influencé d'une part par l'offre en logements, d'autre part par l'importante offre commerciale, récréative et culturelle du centre-ville à l'adresse de catégories démographiques très précises, au style de vie approprié.

Dans le canton de Bâle-Campagne, nous observons un décalage très net entre les communes proches de la ville et les communes rurales. A proximité immédiate de la ville, le taux de ménages d'une personne dépasse $30 \%$; dans les autres communes de l'agglomération et dans les centres du canton il se situe autour de 25-34\%. Dans les communes rurales, par contre, il tombe à moins de $25 \%$. La part des ménages d'une personne montre que de nouveaux types de formes de vie urbaine s'établissent dans l'agglomération à la faveur d'une densification croissante du bâti.

\section{Les types de ménages}

31 L'indication de la taille des ménages ne fournit pas forcément des renseignements précis sur les types de ménages. Parfois l'augmentation du nombre de ménages restreints s'explique d'une part par la part croissante de personnes âgées ou celle de personnes seules, d'autre part par le choix délibéré de mener en tant que personne seule une vie 
individuelle et indépendante. La composition de cette catégorie de ménages est ainsi très hétérogène. Il en est de même des ménages de deux personnes qui comportent, selon la statistique officielle, des couples mariés et non mariés, des ménages monoparentaux et de petites communautés d'habitation.

L'analyse fine de différents types de ménages révèle que des évolutions semblables à celles de la ville de Bâle ont eu lieu dans l'espace proche, toutefois dans des conditions de départ moins prononcées et à une vitesse plus réduite (Tableau 2). Il est cependant étonnant qu'à Bâle-Campagne le taux parental de personnes seules soit plus élevé qu'à Bâle-Ville : 5,5\% par rapport à 4,6\% de l'ensemble des ménages en 1990. Quoique l'on admette souvent que cette forme de ménage soit spécifiquement urbaine, et que l'infrastructure ad hoc y soit plus prononcée, il existe visiblement chez les personnes seules à charge d'enfant(s) une tendance à quitter la ville et donc un taux supérieur de représentation dans l'agglomération même. Un espace disposant d'avantages plus favorables aux enfants, ou offrant des loyers plus modérés, peut également susciter le départ de personnes seules à charge d'enfant(s).

Tableau 2 : Types de ménages dans les Deux-Bâles en 1980 et 1990*, en \%

\begin{tabular}{|l|r|r|r|r|}
\hline \multirow{2}{*}{\multicolumn{1}{|c|}{ Types de ménages }} & \multicolumn{2}{|c|}{ Bâle-Ville } & \multicolumn{2}{c|}{ Bâle-Campagne } \\
\cline { 2 - 5 } & 1980 & 1990 & 1980 & 1990 \\
\hline Pas de famille & 5,1 & 5,1 & 4,6 & 2,8 \\
Personnes seules avec enfant(s) & 4,8 & 4,6 & 5,2 & 5,4 \\
Couples non mariés avec enfant(s) & 0,4 & 0,7 & 0,4 & 9,6 \\
Couples mariés avec enfant(s) & 22,2 & 17,7 & 42,6 & 33,9 \\
Couples non mariés sans enfant(s) & 2,6 & 4,1 & 1,9 & 4,3 \\
Couples sans enfants & 23,0 & 22,4 & 23,1 & 25,3 \\
Personnes seules (« singles ») & 41,8 & 45,2 & 22,1 & 27,2 \\
\hline Total des ménages & 100,0 & 100,0 & 100,0 & 100,0 \\
\hline
\end{tabular}

*Remarque : les données du recensement de 2000 ne seront disponibles qu'en 2003.

Source : Office fédéral de statistique, recensements de 1980 et 1990.

\section{Le niveau de formation}

La statistique suisse ne dispose pas d'indications directes relatives à la répartition des revenus.

Le niveau de formation et la structure des activités fournissent par contre des renseignements sur la situation socio-économique de la population. L'augmentation dans certaines unités spatiales des catégories de population à niveau de formation élevé met en principe l'accent sur la valorisation des quartiers résidentiels concernés.

En ce qui concerne le niveau de formation, les différences entre Bâle-Ville et BâleCampagne sont plutôt minimes en termes de moyenne cantonale. Examinées toutefois à l'échelle microspatiale, les disparités s'avèrent être plus amples. Cette constatation peut être illustrée par les deux exemples suivants: l'un relatif aux habitants n'ayant qu'une formation d'école primaire obligatoire, l'autre propre aux habitants ayant effectué des études supérieures.

36 En 1990, la plupart des personnes sans autre formation que celle de l'école primaire obligatoire habitaient dans la ville de Bâle, plus précisément dans les quartiers 
industriels et ouvriers septentrionaux de Klybeck, Matthäus et Kleinhüningen. Dans le canton de Bâle-Campagne, ce type de population est surtout localisé dans les communes rurales qui ont été marquées pendant longtemps par l'émigration et la stagnation et qui n'ont connu une immigration renforcée que durant les années 1990. Ce phénomène est particulièrement apparent dans les communes du Laufental, où la part de la population ayant achevé des études supérieures diminue au fur et à mesure que l'on s'éloigne de la ville et de l'agglomération immédiatement attenante, en direction des communes rurales. Dans la plupart des communes rurales, le taux des personnes ayant bénéficié d'une formation supérieure est inférieur à $2 \%$. Dans les communes de l'agglomération, il est de 6 à $13 \%$; en ville, il oscille en moyenne autour de 6,5\%. C'est toutefois dans les quartiers résidentiels urbains de Bachletten, Bruderholz, ainsi que dans la (vieille) ville du Grand Bâle que nous observons les taux les plus élevés de l'ensemble de la région, soit 13 à $15 \%$. Les taux sont par contre faibles dans les quartiers industriels et ouvriers du nord de la ville, soit 1,6 à 5,1\%.

Le niveau de formation est donc très varié tant en ville qu'à la campagne. En ville, nous observons une polarisation microspatiale par les niveaux de formation élevé et faible. Dans le canton de Bâle-Campagne, la disparité s'établit entre les communes proches de l'agglomération urbaine et les communes rurales.

\section{E. La structure des activités}

La composition démographique selon les catégories socio-professionnelles est, comme celle du niveau de formation, très peu hétérogène à l'échelle d'une comparaison entre les deux Cantons (fig. 17). Les différences de structure des activités s'accentuent cependant au fur et à mesure de l'approfondissement de l'analyse microspatiale.

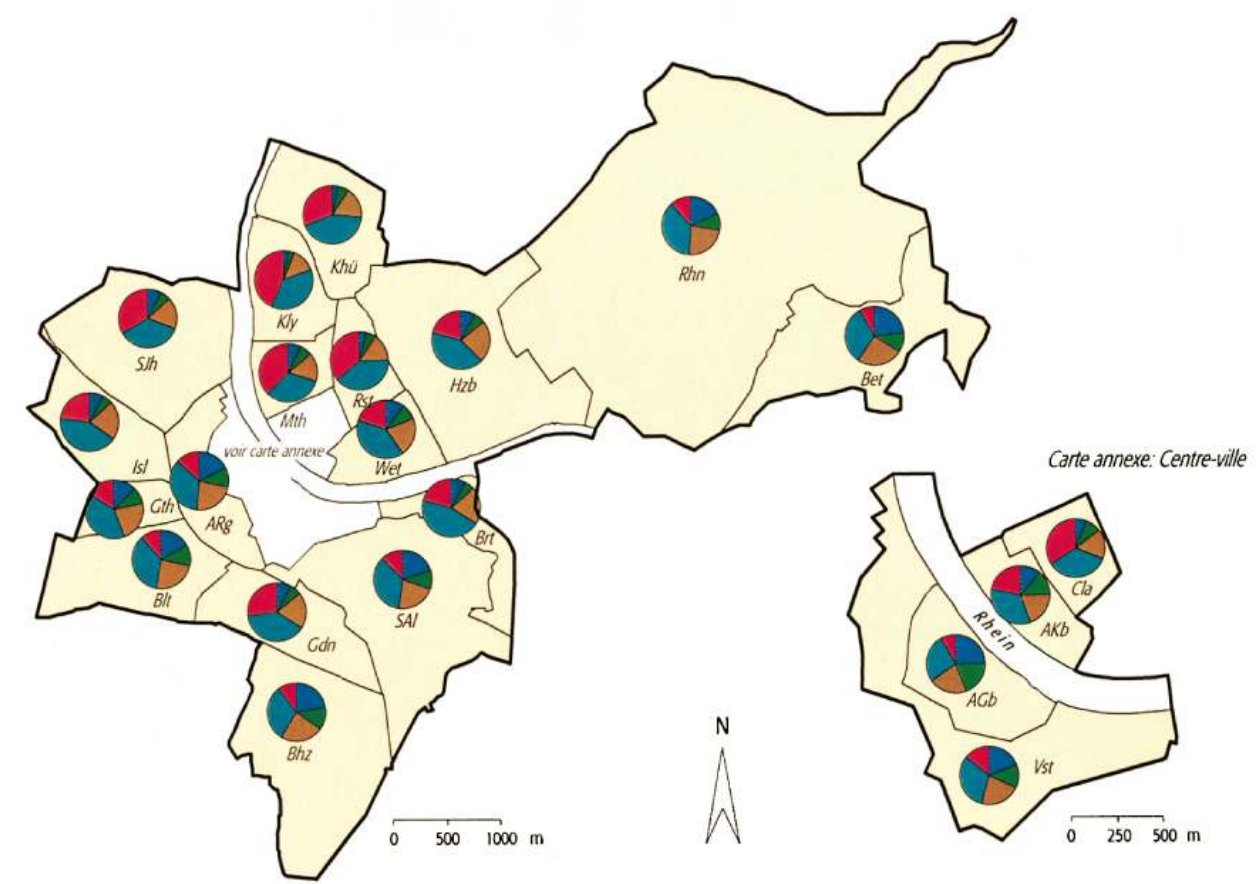


Figure 18 : Population active, selon les catégories socio-professionnelles (sauf les « nonattribuables " et les personnes sans revenu) dans les Deux-Bâles, par circonscriptions, 1990

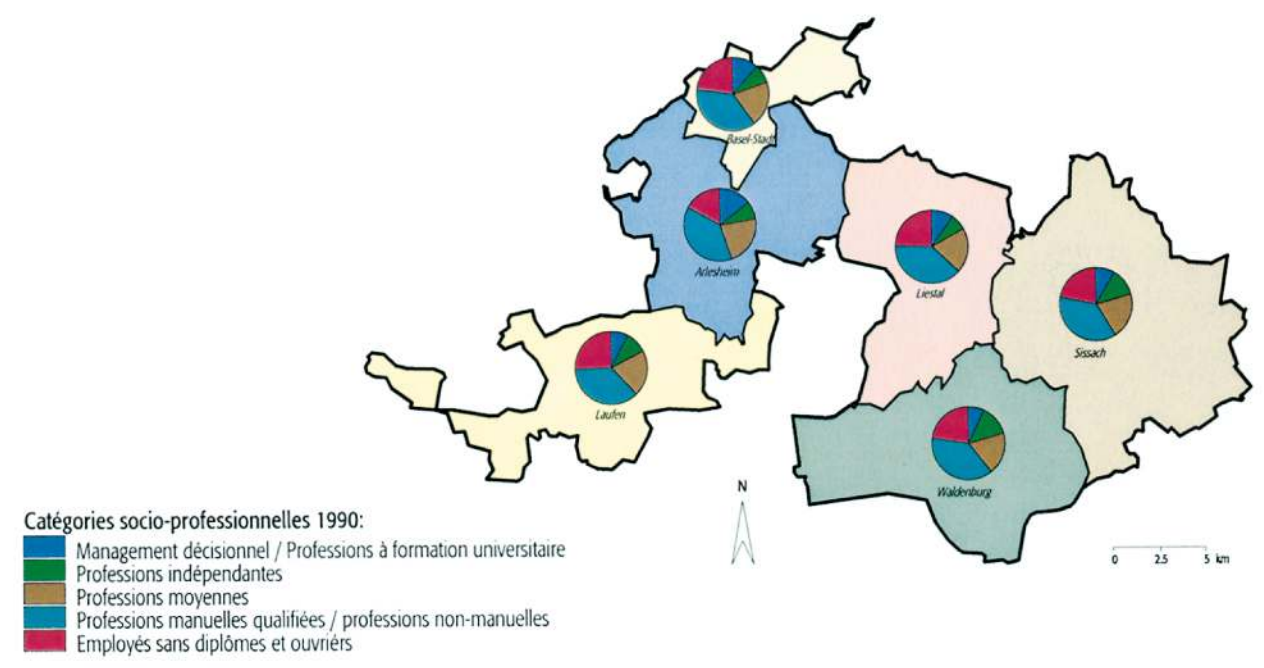

Comme pour le niveau de formation, nous constatons également ici que les quartiers résidentiels de la ville sont très diversifiés. Les taux les plus élevés d'emplois non qualifiés se rapportent aux quartiers industriels et ouvriers du nord de Bâle, depuis Kleinhüningen par Klybeck jusqu'à Sankt-Johann et Rosental. La vieille ville du Grand Bâle, Bruderholz et Sankt-Alban, ainsi que Riehen et Bettingen, atteignent les taux les plus élevés en ce qui concerne les cadres supérieurs et les personnes à formation universitaire. La plupart des membres des professions libérales et indépendantes habitent dans la vieille ville de Grand Bâle et de Petit-Bâle.

Dans le canton de Bâle-Campagne, la circonscription d'Arlesheim, proche de l'agglomération urbaine, se distingue nettement des autres circonscriptions (fig. 18). Elle comporte un taux relativement plus élevé de résidents à formation supérieure et de cadres supérieurs. Ce taux - 14,8 \% - se situe même au-dessus de celui propre au canton de Bâle-Ville. Il contribue au fait que le taux d'habitants à formation supérieure et de cadres supérieurs actifs dans le management est dans l'ensemble plus élevé dans le canton de Bâle-Campagne que dans celui de Bâle-Ville. A Arlesheim, le taux d'habitants non qualifiés se situe en conséquence nettement au-dessous de celui observé dans les autres circonscriptions.

41 Comparée aux Etats voisins, la Suisse ne pose pas de problème social majeur en matière de chômage. Le taux de chômage des Deux-Bâles, qui a encore atteint 3,9 \% en 1998, ne s'est plus élevé en 2000 qu'à $1,7 \%$. La même année, Bâle-Ville n'a enregistré qu'un taux de $1,8 \%$ et Bâle-Campagne de $1,6 \%$.

La population étrangère connaît toutefois un taux de chômage supérieur à la moyenne. Cela signifie pour les communes concernées par ce phénomène une diminution des ressources fiscales et un surcroît de dépenses par des transferts publics.

Les recettes fiscales des différentes communes constituent aussi un indicateur destiné à appréhender la structure des revenus de la population. Dans le domaine des impôts fédéraux directs, les communes de l'agglomération bâloise atteignent les taux les plus élevés. La ville de Bâle toutefois n'a enregistré en 1991/1992 qu'un revenu fiscal/habitant de 850 FS, inférieur à la moyenne des communes des Deux-Bâles, soit 915 FS. 

certains types d'évolution. A côté du Grand-Bâle qui représente un cas particulier par sa structure démographique propre aux grandes villes, certaines communes qui se situent à proximité immédiate sont caractérisées par un taux élevé de résidents appartenant aux couches moyennes de la population, composées principalement de couples ou de familles à revenu élevé. Les autres communes de l'agglomération bâloise et quelques communes certes plus éloignées, mais localisées le long des axes majeurs de communications, ainsi que quelques centres ruraux correspondent à peu près à la moyenne cantonale en matière de structure démographique. D’autres communes, plus éloignées encore, ont connu un important solde migratoire positif au cours des dernières années. La plupart d'entre elles ont conservé un cachet rural bien marqué ; elles enregistrent cependant des taux d'immigration élevés et connaissent les premiers symptômes d'une restructuration du village traditionnel. Les petites communes rurales ne sont plus qu'une exception dans le canton de Bâle-Campagne (fig. 1 et 3). vue de sa composition démographique. L'imposante rénovation de son bâti historique a attiré essentiellement de jeunes Suisses, à formation poussée et à revenu confortable, très fréquemment sous la forme de ménages d'une personne. Les quartiers résidentiels localisés au tour du centre-ville du Grand-Bâle (fig. 2) n'ont pas de population à problèmes et reflètent plutôt le profil moyen de la ville. Le grand nombre de petits logements y explique le taux quelque peu élevé de ménages à une personne.

quartiers industriels et tertiaires du nord de la ville, au taux élevé de population étrangère, se composent surtout d'habitants relativement jeunes, dont le niveau de formation est plus ou moins bon ou dont la formation est en cours. Ce groupe de résidents habite Klybeck, Kleinhüningen, Sankt-Johann, Matthäus, Rosental, Clara, voire le quartier de Gundeldingen. Les quartiers résidentiels à la périphérie de la ville sont le propre des couches moyennes et aisées de la population; c'est le cas de Hirzbrunnen, Sankt-Alban, Bruderholz et Bachletten. La part des étrangers y est faible, tandis que le niveau de 
formation est élevé. Les ménages à une personne y sont rares et la proportion de population âgée est supérieure à la moyenne générale.

\section{III. État des lieux et perspectives}

Nous venons de constater qu'en matière démographique, la ville de Bâle est également une région de grande ville aux fractures socio-spatiales très différenciées dans ses quartiers intra-urbains comme dans les communes suburbaines. L'analyse microspatiale a permis d'observer de façon croissante l'émergence d'un modèle de ségrégation à l'échelle des quartiers. Ceux-ci se distinguent en effet partiellement les uns des autres par la composition de leur population résidente. Les différences apparaissent encore davantage au niveau des îlots d'habitation (Eder, 2001).

51 L'une des répercussions des pertes démographiques du noyau urbain est la baisse grandissante des recettes fiscales. Comme les habitants à revenu moyen et supérieur ont tendance à quitter ce noyau pour aller s'établir dans l'espace environnant, l'arrivée de catégories de population au revenu modeste ne peut guère compenser le manque à gagner fiscal. Les partants donnent comme motivation de leur départ l'insuffisante qualité environnementale, le manque d'équipements pour enfants, l'insuffisante offre résidentielle en ville, la pénurie en espaces verts et parcs (Kampschulte/ Schneider-Sliwa, 1999). Les arrivants motivent leur venue par les trop grandes distances par rapport au noyau urbain, partiellement aussi par l'insuffisante accessibilité à ce noyau, lequel constitue pour eux le lieu de travail. Ces mouvements de population ne sont pas uniquement suscités par la diminution du nombre d'habitants de Bâle-Ville, mais aussi par les flux de trafic grandissants en direction de Bâle.

Les noyaux urbains de diverses agglomérations suisses, dont celui de Bâle, sont pénalisés par le fait que, parallèlement à la perte de résidents au pouvoir d'achat élevé, les exigences en matière de prestations publiques ne diminuent pas autant que les recettes urbaines. Les fortes dépenses inhérentes au service public doivent être financées à partir d'un produit fiscal en voie de régression (Odermatt, 1999; Wehrli-Schindler, 1995). Les villes doivent à présent faire face à des problèmes dont elles ne sont pas les seules responsables et dont l'origine est plus complexe que la situation illustrée dans les années 1990 sur les tréteaux de la « Zürcher Drogenszene » (internationalement célèbre) dans la pièce intitulée "Lieu d'apparition, de problématique et de décision " ("Entstehungs-, Problem- und Entschei-dungsort ", Wehrli-Schindler, 1995). La population de l'agglomération urbaine attend simultanément de la part de la ville une offre culturelle et récréative variée et de grande envergure, ainsi que des possibilités de formation. Les noyaux urbains doivent en conséquence faire face à des problèmes financiers croissants.

En réaction à ces évolutions, la discussion sur les questions soulevées s'est ouverte dans plusieurs villes suisses. Ce n'est pas qu'à Berne que se précisent des revendications favorables à une fusion du noyau urbain avec les communes environnantes au solide pouvoir financier (Basler Zeitung du 7.8.2001). A Bâle aussi, le départ hors du noyau urbain au cours des dernières années de groupes aux revenus élevés et la perte consécutive de "bons" redevables fiscaux ont contribué à susciter une importante discussion publique sur ce thème. Dans le cadre du projet «Werkstadt Basel » («Atelier Bâle »), financé par le gouvernement de la Ville de Bâle, ces préoccupations ont été reprises; des stratégies destinées à contrer les mouvements en cours ont été élaborées selon des processus impliquant de multiples participations. En 1999 a été adopté le 
«Programme d'action pour le développement urbain de Bâle» («Aktionsprogramm Stadtentwicklung Basel »), un schéma directeur pour la politique urbaine des prochaines années. L'un des objectifs majeurs de ce programme d'action consiste à privilégier la construction de logements spacieux et bien équipés, de même que la valorisation de l'espace résidentiel en vue de "reconquérir " les redevables fiscaux appartenant aux catégories médianes de la population (Wiener, 2001 ; Regierungsrat Basel-Stadt, 1999).

L'arrivée de groupes de résidents à niveau de revenu élevé contribuerait en effet à régler ce problème déterminant posé aujourd'hui aux villes. Indépendamment de la recherche d'une solution pour cette question capitale, il convient en outre de ne pas négliger d'autres aspects :

- pour réussir à redevenir attractives pour les catégories à revenu cossu, les villes, en dotant certains quartiers de logements de grande qualité et coûteux, chassent des habitants modestes dans la mesure où ce genre de restructuration entraîne aussi la mutation des structures démographiques (Buchmüller et al., 2000; Wehrli-Schindler, 1995 ; Schwendener, 2001). Le renforcement de la demande en localisations intra-urbaines risque notamment de déclencher en ville des processus de "gentrification" et d'élimination sociale, surtout lorsque la rénovation fait monter les loyers à tel point que la population en place ne parvient plus à y faire face ;

56 - la «valorisation" devrait ainsi être comprise dans le sens où les déficits résidentiels existant en matière infrastructurelle et de bâti donnent lieu à suppression, mais dans le respect des intérêts et aspirations des personnes vivant dans le quartier. Les habitants actuels doivent pouvoir y demeurer eux aussi et s'y sentir à l'aise. Pour contribuer à alléger financièrement le poids des charges qui grève les noyaux urbains, il conviendrait de parler, plutôt que d'une "valorisation » de la ville, d'une modification du système de compensation financière intercommunale et cantonale, ainsi que de la recherche d'une répartition fiscale adéquate entre le lieu d'habitation et le lieu de travail (Bundesamt für Raumplanung, 1998) ;

57 - les processus évoqués ci-dessus ne sont nullement propres à Bâle. On peut d'ailleurs se demander de manière croissante si les mesures prises pour les contrer sont vraiment à même de permettre de maîtriser la situation (Neff, 2001). Le seul recours à la solution de l'apport de «bons » redevables fiscaux n'est certainement pas en mesure de régler les problèmes d'une grande ville placée face aux perspectives croissantes d'une mise en réseaux internationale, de la globalisation et du renforcement de la compétition intervilles.

58 Toute politique de développement urbain devrait donc être fondée en premier lieu sur les préoccupations relatives à la population en place, parallèlement à celle concernant l'apport de résidents à niveau de revenu élevé. A cet effet, il faut tenter en premier lieu de rendre la quartier concerné suffisamment attractif pour les habitants actuels, en vue d'améliorer leurs propres conditions de vie. La mise en place de «Werkstadt Basel », un processus de dialogue élargi à l'ensemble de la ville, est déjà à ce sujet un bon départ. A l'étranger également certaines grandes villes ont adopté entre-temps des actions destinées à promouvoir dans les quartiers défavorisés un développement urbain socialement "soutenable ", par le truchement d'un management de quartier adapté. C'est le cas en Allemagne, à l'échelle nationale, avec le programme fédéral intitulé "Ville sociale", particulièrement orienté vers les "Quartiers aux besoins de développement spécifiques ». La pratique de «Werkstadt Basel » à Bâle a abouti à des approches analogues. Dans ce processus bâlois, le rôle d'associations indépendantes de quartier, qui s'appuient sur une 
tradition presque centenaire, est d'ailleurs plus important que celui de l'administration publique, dès lors qu'il s'agit de favoriser la rencontre des diverses composantes culturelles et d'améliorer les conditions de vie dans le quartier. Les potentialités de l'administration publique en ce qui concerne l'ampleur des problèmes soulevés par ce chantier sont encore loin d'être épuisées.

\section{BIBLIOGRAPHIE}

BASLER ZEITUNG (2002). - Die Nordwestschweiz wächst in der Peripherie ; p. 2. Volkszählung entlarvt Visionen der Raumplanung als Illusion ; p.3.

BuchmülleR L., DonAld A. KelleR, косH M., SCHUMACHER F., SELlE K. (2000). - Planen Projekte Stadt? Weitere Verständigungen über den Wandel in der Planung. In : DISP 141, S. 55-59.

BUNDESAMT FÜR RAUMPLANUNG (Hrsg.) (1998). - Erneuerung und Stärkung der Städte. Auswirkungen der verschiedenen Bundespolitiken. Eine Diskussionsgrundlage. Verfasst von INFRAS Zürich und Bern. Bern : EDMZ.

EDER S. (2001). - Städtische Sozialstrukturen und residentielle Segregationsmuster am Beispiel Basel-Stadt. In : Geographica Helvetiva, Heft 4/2001.

KAMPSCHUlTE A., SCHNEIDER-SLIWA R. (1999). - Das Image von Basel. Steuerungsinstru-ment für die Stadtentwicklung? Basler Feldbuch, Band 16. Basel.

NEFF M. (2001). - Standortpoker. In : Wiener (Hrsg. ), S. 106-107.

Odermatt A. (1999). - Räumlich-soziale Entmischung und die Finanzkrise der Kernstädte - das Beispiel Zürich. In : GH, Jg. 54 Heft 1, S. 18-28.

REGIERUNGSRAT DES KANTONS BASEL-STADT (1999). - Aktionsprogramm Stadtentwicklung

Basel. Ergebnisse der Werkstadt Basel. Basel.

SCHNEIDER-SLIWA R. et al. (1999). - Bevölkerungsstruktur und Bevölkerungsdynamik beider Basel. Basel : Statistisches Amt des Kantons Basel-Stadt.

SCHWENDENER P. (2001). - Zahlen zählen. In : Wiener (Hrsg. ), S. 64-65.

Wiener D. (Hrsg.) (2001). - Wir sind die Stadt. Das Beispiel Werkstadt Basel. Basel : Christoph Merian Verlag.

WeHRLI-SCHINDLER B. (1995). - Lebenswelt Stadt. Berichte zur Lebenssituation in Schweizer Städten. Teilsynthese des Nationalen Forschungsprogramms 25 "Stadt und Verkehr". Zürich : vdf.

WiMMER A. (2000). - Städtevergleich, Netzwerkanalyse und Schlussfolgerungen. Teil des Schlussberichtes für das Forschungsprojekt "Integration - Segregation : Interkulturelle" Beziehungen in Basel, Bern und Zürich. 


\section{RÉSUMÉS}

La mutation de la société, la restructuration économique et les migrations internationales ont modifié la structure démographique de nombreuses villes d'Europe occidentale. Elles ont suscité des modèles variés en matière de différenciations et de fractures socio-spatiales. L'article s'appuie sur l'exemple de la région de Bâle pour y faire ressortir les transformations des structures démographiques et sociales intervenues au cours des dernières décennies. Les bases statistiques de l'étude sont fournies par les résultats des recensements de la population suisse, ainsi que par des enquêtes complémentaires effectuées par l'office de statistique cantonal de Bâle-ville et Bâle-campagne. Le noyau urbain de Bâle perd de façon continue des habitants et cette mobilité est sélective de façon croissante. La région bâloise est également soumise au processus de fragmentation micro-spatiale, aux fractures socio-spatiales et à l'hétérogénéisation ethnique de la structure démographique. Cette évolution a conduit à une réorientation de la politique de développement urbain de Bâle en vue du freinage du mouvement d'émigration des catégories de la population à revenus élevés.

Societal change, economic restructuring and international migration have been changing the demographic structure of many cities in Western Europe, leading to new patterns of segregation. This paper presents the intercensal changes in the demographic and social structure of the Cantons of Basle based on the Swiss Census and complementary data. The results show that the City Canton of Basle has been losing population steadily to the surrounding Canton of BasleCountry and that this has been socially selective. As a result, both cantons of Basle display increasingly small-scale social fragmentation, social and demographic segregation and ethnic heterogenity. This has been of concern to urban policy makers in the City Canton of Basle, leading to countermeasures with a focus on keeping « good tax payers » in the City of Basle.

Gesellschaftlicher Wandel, ökonomische Umstrukturierung und internationale Migration haben die Bevölkerungsstruktur zahlreicher westeuropäischer Städte verändert und vielfältige Muster neuer sozialer Ausdifferenzierung entstehen lassen. Der vorliegende Beitrag stellt die Veränderungen der Bevölkerungs- und Sozialstruktur der letzten Jahrzehnte für die Region Basel dar. Die Grundlage hierfür bilden die Ergebnisse der Schweizer Volkszählungen sowie ergänzende Erhebungen der kantonalen Statistik für Basel-Stadt und Basel-Landschaft. Die Ergebnisse zeigen, dass die Kernstadt Basel sukzessive Einwohner verliert, und dass diese Mobilität zunehmend sozial selektiv ist. Auch in der Region Basel ist daher eine kleinräumige Fragmentierung, soziodemographische Ausdifferenzierung und ethnische Heterogenisierung der Bevölkerungsstruktur festzustellen, was zu einer Neuorientierung der Stadtentwicklungspolitik von Basel und einer Gegensteuerung zur Abwanderung einkommensstärkerer Bevölkerungsgruppen geführt hat.

\section{INDEX}

Mots-clés : Bâle, différenciation et fractures socio-spatiales, évolution de la population, ségrégation

Keywords : Basle, population change, social fragmentation

Schlüsselwörter : Basel., Bevölkerungsentwicklung, Soziale Ausdifferenzierung 


\section{AUTEURS}

\section{RITA SCHNEIDER-SLIWA}

Institut de géographie de l'Université de Bâle - Klingelbergstrasse 16 CH- 4056 Basel Rita.Schneider-Sliwa@unibas.ch

\section{ANDREA KAMPSCHULTE}

Institut de géographie de l'Université de Bâle - Klingelbergstrasse 16 CH- 4056 Basel

\section{DANIELA ZUNZER}

Institut de géographie de l'Université de Bâle - Klingelbergstrasse 16 CH- 4056 Basel 